Published in final edited form as:

Compr Psychiatry. 2014 April ; 55(3): 526-533. doi:10.1016/j.comppsych.2013.10.007.

\title{
Comorbidity Between Major Depression and Alcohol Use Disorder From Adolescence to Adulthood
}

\author{
Frédéric N. Brière, $\mathrm{PhD}^{1,2}$, Paul Rohde, $\mathrm{PhD}^{3}$, John R. Seeley, $\mathrm{PhD}^{3}$, Daniel Klein, $\mathrm{PhD}^{4}$, \\ and Peter M. Lewinsohn, $\mathrm{PhD}^{3}$ \\ ${ }^{1}$ Université de Montréal, Québec, Canada \\ ${ }^{2}$ School Environment Research Group (SERG), Québec, Canada \\ ${ }^{3}$ Oregon Research Institute, Oregon, USA \\ ${ }^{4}$ Stony Brook University, NY, USA
}

\section{Abstract}

Background-Limited information exists regarding the long-term development of comorbidity between Major Depressive Disorder (MDD) and Alcohol Use Disorder (AUD; abuse/dependence). Using a representative prospective study, we examine multiple aspects pertaining to MDD+AUD comorbidity, with a focus on the relation between disorders across periods (adolescence, early adulthood, adulthood) and cumulative impairments by age 30 .

Method-816 participants were diagnostically interviewed at ages 16, 17, 24, and 30 .

Results-Rates of comorbid MDD+AUD were low in adolescence (2\%), but increased in early adulthood (10\%) and adulthood (7\%). Rates of cumulative comorbidity were elevated (21\%). Most individuals with a history of MDD or AUD had the other disorder, except for women with MDD. Prospectively, adolescent AUD predicted early adult MDD, while early adult MDD predicted adult AUD. Compared to pure disorders, MDD+AUD was associated with higher risk of alcohol dependence, suicide attempt, lower global functioning, and life dissatisfaction.

Conclusions-Lifetime rates of comorbid MDD+AUD were considerably higher than in crosssectional studies. Comorbidity was partly explained by bidirectional and developmentally-specific associations and predicted selected rather than generalized impairments. Clinically, our findings emphasize the need to always carefully assess comorbidity in patients with MDD or AUD, taking into account concurrency and developmental timing.

\section{Keywords}

Comorbidity; Major Depression; Alcohol Use Disorder; Longitudinal; Adolescence; Adulthood

\footnotetext{
(C) 2013 Elsevier Inc. All rights reserved.

Correspondence: Frédéric N. Brière, Université de Montréal, C.P. 6128, succursale Centre-ville, Montréal, Québec, Canada, H3C 3J7.Telephone: 514-452-0332. (frederic.naultbriere@umontreal.ca, permission granted to publish this address).

Publisher's Disclaimer: This is a PDF file of an unedited manuscript that has been accepted for publication. As a service to our customers we are providing this early version of the manuscript. The manuscript will undergo copyediting, typesetting, and review of the resulting proof before it is published in its final citable form. Please note that during the production process errors may be discovered which could affect the content, and all legal disclaimers that apply to the journal pertain.
} 


\section{Introduction}

Comorbidity between Major Depressive Disorder (MDD) and Alcohol Use Disorder (AUD; abuse or dependence) represents one of the most prevalent and disabling psychiatric combinations in adolescence and adulthood. In the last decades, clinical and epidemiological studies have documented the rates and characteristics of MDD+AUD comorbidity, especially in adulthood (1-5). Although longitudinal studies have investigated associations between the two disorders, none has comprehensively examined the development and implications of comorbidity from adolescence to adulthood. Here, we use a prospective sample of participants followed to age 30 to investigate several key issues of comorbidity (6) applying to MDD+AUD.

Our first focus is on the relation between the two disorders. Using two complimentary angles, we analyze the temporal ordering and predictions between MDD and AUD across key developmental periods. Evidence on temporal ordering from retrospective studies has been mixed, with studies reporting MDD to most often precede AUD $(1,7,8)$, AUD to most often predece MDD $(9)$ or no clear ordering $(2,10)$. Similarly, predictions between disorders have been conflicting. Prospective studies have found MDD to predict AUD $(8,11)$, AUD to predict MDD $(12,13)$, bidirectional relations $(9,14,15)$ and no association between the two disorders after controlling for confounders (16). A key limitation of the literature is that most evidence comes from adult samples of wide age ranges $(8,9,11,14-16)$, making it difficult to determine whether associations vary across developmental periods.

Our second focus is on "cumulative comorbidity", which indexes lifetime rather than point co-occurrence of disorders (17). We examine the implications of having lifetime patterns with or without overlapping disorders (concurrent vs. successive comorbidity) (6). Very little information is available to document these types of comorbidity. We also investigate clinical and psychosocial impairments associated with lifetime comorbid MDD+AUD compared to pure MDD and AUD. Greater impairments have typically been reported in comorbid individuals (e.g., more persistent and severe disorders, suicide risk, lower social adjustment) $(1,4,18-20)$, but other studies found no poorer or less adverse outcomes of comorbidity, notably lower AUD severity compared to pure AUD $(21,22)$. We also consider whether impairments vary as a function of both concurrency or temporal ordering (MDD-first vs. AUD-first). To our knowledge, no prospective study has previously examined cumulative impairments of MDD+AUD in adulthood.

We use data from the Oregon Adolescent Depression Project (OADP) (23) to address these questions. The OADP is unique in combining 4 prospective assessment waves from adolescence to adulthood paired with retrospective recall between assessments, providing diagnostic coverage up to age 30 . This design compares favorably to most prospective studies, which have more limited coverage, as well as retrospective studies, which are impacted by long-term recall bias. In keeping with the new DSM-5 (24), we investigate AUD as a single disorder, rather than abuse and dependence as separate disorders. We do, however, consider alcohol dependence as a marker of AUD severity. 


\section{Method}

\section{Participants}

OADP participants were followed at four time points (23). The sample was randomly selected from nine high schools representative of western Oregon. After complete description of the study to the participants, written informed consent was obtained. Ethics approval was granted by the Oregon Research Institute. At T1, 1709 adolescents were administered a diagnostic interview and questionnaires at an average age of 16.6 years $(S D=1.2)$. The sample was approximately equally-divided by gender (53\% women) and mostly White (91\%). Participants were invited to complete a second assessment approximately one year later. At T2, 1507 participants (88\% of T1) returned for a second assessment. A subset of participants was invited to a third assessment at age 24 (T3): all participants with a history of psychopathology $(N=555)$ and a randomly selected subset of participants with no history of mental disorder $(N=386)$; 941 participants (85\% of those invited) completed T3 assessments. All T3 participants were invited for a fourth wave at age 30; 816 participants (87\% of T3) completed assessments at $\mathrm{T} 4$. The present study uses OADP participants who completed assessments up to T4 (59\% women; 89\% White). Differences between persons who continued and discontinued participation were overall small (25). Correlates of discontinuation were male gender, cigarette and substance use, a history of a disruptive behavior disorder, lower socioeconomic status, and fewer persons living at home during adolescence.

\section{Measures}

Diagnoses of MDD and AUD, as well anxiety disorders, disruptive behavior disorders (DBD), and other substance use disorders (SUD; excluding tobacco dependence) were obtained from standardized diagnostic interviews. T1-T3 interviews used a version of the Schedule for Affective Disorders and Schizophrenia for School-Age Children (K-SADS) (26), combining features of the Epidemiologic and Present Episode versions. T4 used the Structured Clinical Interview for DSM-IV (SCID) (27). Diagnoses were based on DSM-III$\mathrm{R}$ criteria at $\mathrm{T} 1$ and $\mathrm{T} 2$ and DSM-IV criteria at $\mathrm{T} 3$ and $\mathrm{T} 4$. The $\mathrm{T} 1$ interview recorded lifetime disorders from the age of 5. In T2-T4 interviews, the Longitudinal Interval FollowUp Evaluation (LIFE) (28) assessed psychopathology since the previous interview.

Clinical characteristics were collected from diagnostic interviews. Disorder duration was calculated as the cumulative duration of MDD and AUD episodes in weeks. Disorder severity was defined as the lifetime occurrence of severe depression for MDD based on the DSM episode specifier and lifetime occurrence of alcohol dependence as opposed to abuse only for AUD. By T4, 121 AUD participants had a lifetime alcohol abuse diagnosis only (42.9\%), 113 had a lifetime dependence diagnosis only (40.1\%), and 48 had both lifetime diagnoses (17.0\%). Lifetime suicide attempts and mental health treatment were assessed using relevant sections of the K-SADS and SCID. Psychosocial outcomes at T4 included marital status ( $1=$ not married; $0=$ married), parental status ( $1=$ never parent; $0=$ parent), years of schooling completed, weeks of unemployment in the past year, annual household income, global functioning (GAF; DSM-III-R/-IV), self-rated physical health (4-item scale; $\alpha=0.50$ ), 
life satisfaction ( 15 -item scale; $\alpha=0.89$ ), and past-year risky sexual behavior ( $1=$ any report of risky sex on 9 items; $0=$ none).

\section{Statistical Analyses}

We examined comorbidity between MDD and AUD in three developmental periods: Adolescence (two years prior to T1 up to and including T2); Early Adulthood (immediately after T2 up to and including T3); and Adulthood (immediately after T3 up to and including T4). We used path analysis to evaluate associations between MDD and AUD across periods. We specified a cross-lagged model with 1) cross-sectional associations between MDD and AUD in each period, 2) continuity associations for each disorder between adjacent periods, and 3) prospective associations from one disorder to the other in adjacent periods. Anxiety disorders, Disruptive behavior disorders, and other Substance use disorders were used as controls in adolescence. We used probit regressions to evaluate associations between disorders over time in Mplus 6.21 (29). Acceptable model fit (CFI and TLI > $=0.90$; RMSEA $<=0.08$ (30) was validated before examining individual paths. We examined differences between comorbid and pure disorders on clinical and psychosocial impairments using linear or logistic regression adjusting for sex, anxiety disorders, DBD, and other SUD by T4. We evaluated differences as a function of temporal ordering, concurrency, or the ordering x concurrency interaction separately. Temporal ordering was coded as MDD-First, AUD-first or simultaneous onset based on the month of onset of the first lifetime MDD and AUD episodes. Concurrent comorbidity was coded as any history of overlapping MDD and AUD episodes by age 30, while successive comorbididy was coded as no history of overlapping MDD and AUD episodes by age 30. We used T3 sampling weights in all analyses.

\section{Results}

\section{Period and Cumulative Prevalence}

Table 1 presents the prevalence of pure and comorbid MDD and AUD by developmental period. Rates of pure MDD were already high in adolescence and remained relatively constant at approximately $20 \%$ over each period. Rates of pure AUD and comorbid MDD +AUD were low (below 5\%) in adolescence, but escalated to 10-21\% in early adulthood, before leveling down to 5-18\% in adulthood. Patterns of MDD and AUD differed by sex in all periods (Chi square, $p<.001$ ): pure MDD was more prevalent in women, whereas pure AUD and no disorder were more prevalent in men. The only exception was pure AUD in adolescence, which did not differ by sex. Interestingly, rates of comorbid MDD+AUD did not differ by sex in any period.

The bottom portion of Table 1 shows the cumulative rates of pure and comorbid MDD and AUD by T4. Comorbid MDD+AUD remained less prevalent than pure MDD by age 30, but was more prevalent than pure AUD. Consistent with developmental periods, rates differed by sex (Chi square, $p<.001$ ), with higher rates of pure MDD in women and higher rates of no disorder and pure AUD in men. Cumulatively, $40 \%$ of participants with lifetime MDD had a history of AUD and 59\% of individuals with lifetime AUD had a history of MDD. The 
cumulative association between disorders was moderate in magnitude $(\mathrm{OR}=1.6$, $95 \% \mathrm{CI}=1.2-2.1)$ and did not differ by sex.

\section{Comorbidity as A Function of Temporal Ordering and Concurrency}

We next examined temporal ordering and concurrency of MDD and AUD in individuals with cumulative comorbidity by T4. MDD occurred before AUD in 57\% of individuals with comorbid disorders. AUD occurred first in the remaining $41 \%$ of cases, except for rare instances of simultaneous onset (2\%). Cumulative MDD+AUD comorbidity was equally divided between concurrent and successive comorbidity (51\% v. 49\%). Figure 1 depicts the proportion of comorbid subtypes as a function of lifetime temporal ordering and concurrency.

We also investigated temporal ordering specifically in concurrent MDD+AUD. We considered the first concurrent episode in individuals with multiple episodes to avoid dependency. In contrast to lifetime temporal ordering, AUD occurred first in 57\% of concurrent episodes; MDD occurred first in $23 \%$ of concurrent episodes and simultaneous onset represented the remaining $20 \%$ of concurrent episodes. In the majority of cases (62\%), concurrent episodes developed after one or more previous non-concurrent episode(s) of MDD or AUD. Thirty percent of concurrent episodes were preceded by MDD only, 11\% were preceded by AUD only, and $21 \%$ were preceded by non-concurrent episodes of both MDD and AUD.

\section{Associations Between Disorders Across Periods}

Figure 2 presents the cross-sectional and prospective associations between MDD and AUD over time, adjusting for adolescent anxiety disorder, DBD, and other SUD. MDD and AUD were moderately stable over time, with two significant interactions by gender. Adolescent AUD was predictive of early adult AUD for women but not men, and early adult MDD was more predictive of adult MDD in men than women, though continuity for both sexes was significant. MDD and AUD were associated in every period. AUD in adolescence was predictive of MDD in early adulthood, but not from early adulthood to adulthood. Conversely, MDD in early adulthood was predictive of AUD in adulthood, but not from adolescence to early adulthood. Cross-sectional and prospective associations between the two disorders did not vary by sex.

\section{Cumulative Lifetime Clinical Characteristics}

Table 2 presents analyses comparing lifetime clinical characteristics in pure and comorbid MDD and AUD, adjusting for sex and cumulative anxiety disorder, DBD, and other SUD. Comorbidity was not associated with greater MDD or AUD duration or depression severity. Conversely, comorbid MDD+AUD was associated with greater AUD severity (i.e., alcohol dependence). Two-thirds of participants with comorbid MDD+AUD had alcohol dependence compared to less than half of pure AUD participants. A similar pattern emerged for mental health treatment: individuals with comorbid MDD+AUD did not differ from individuals with pure MDD, but were approximately three times more likely to receive treatment than individuals with pure AUD. Comorbid MDD+AUD was associated with 
greater odds of lifetime suicide attempt than both pure MDD, by twofold, and pure AUD, by sevenfold.

We also examined clinical characteristics in comorbid subtypes based on temporal ordering and concurrency. Concurrency and temporal ordering interacted in predicting MDD duration $(B=90.6,95 \% \mathrm{CI}=22.8-158.4)$. Follow-up analyses indicated that participants with concurrent MDD+AUD and MDD-First had strikingly longer cumulative lifetime duration of MDD episodes (median=95.5 weeks) than participants in all other comorbid groups, as well as the pure MDD group (median duration for all other groups=15.9-24.0 weeks). Conversely, duration of AUD episodes varied as a function of concurrency but not temporal ordering. Follow-up analyses indicated that duration of AUD episodes was higher in the concurrent group (median=182.0 weeks) than in the successive group (median=91.6 weeks) ( $\mathrm{B}=86.3,95 \% \mathrm{CI}=41.5-131.1$ ), but not in the pure AUD group (median=132.0 weeks). Duration was higher in the pure AUD group than the successive group $(\mathrm{B}=57.0$, 95\% CI=10.8-103.2). MDD and AUD severity did not differ by temporal ordering or concurrency. Finally, risk of suicide attempt varied as a function of temporal ordering. Odds of suicide attempt were greater in MDD-First (34.5\%) than AUD-First (19.2\%) (OR=2.2, 95\% CI=1.0-4.7), pure MDD (13.3\%) $(\mathrm{OR}=3.4,95 \% \mathrm{CI}=2.0-6.0)$, and pure AUD (3.7\%) $(\mathrm{OR}=9.4,95 \% \mathrm{CI}=3.2-27.7)$. Odds of suicide attempt were also higher for the AUD-First group compared to pure AUD ( $\mathrm{OR}=4.1,95 \% \mathrm{CI}=1.3-13.3)$.

\section{Psychosocial Outcomes At Age 30}

Table 3 shows the psychosocial outcomes at age 30 (T4) of lifetime pure and comorbid MDD and AUD, adjusting for sex and lifetime anxiety disorder, DBD, and other SUD. Comorbid MDD+AUD was predictive of lower global functioning and life satisfaction than both pure MDD and pure AUD. MDD+AUD was also associated with greater odds of being unmarried compared to pure MDD, but not pure AUD. MDD+AUD comorbidity did not differ from pure disorders on other psychosocial outcomes. Last, a comorbidity $\times$ gender interaction $(\mathrm{B}=0.08,95 \% \mathrm{CI}=.001-0.15)$ indicated that MDD+AUD was associated with lower unemployment than pure MDD in men $(\mathrm{B}=-0.08 .95 \% \mathrm{CI}=-0.15--0.02)$, but not women.

Examination of comorbid subtypes revealed few differences. Compared to successive comorbidity, concurrent comorbidity was associated with lower global functioning ( $\mathrm{B}=-3.7$, 95\% CI=-7.3--0.03). Follow-up analyses indicated that both concurrent $(\mathrm{B}=-6.9$, $95 \% \mathrm{CI}=-10.2--3.6)$ and successive $(\mathrm{B}=-3.4,95 \% \mathrm{CI}=-6.7--0.1)$ comorbidity were associated with lower functioning than pure AUD, but only concurrent comorbidity was associated with lower functioning than pure $\mathrm{MDD}(\mathrm{B}=-6.6,95 \% \mathrm{CI}=-9.6--3.6)$. Concurrent comorbidity was also associated with higher odds of risky sexual behavior than successive comorbidity $(\mathrm{OR}=2.4,95 \% \mathrm{CI}=1.2-4.8)$. Follow-up analyses indicated that concurrent, but not successive, comorbidity increased the odds of risky sex relative to pure MDD $(\mathrm{OR}=2.1$, 95\% CI $=1.1-3.8$ ). 


\section{Discussion}

To our knowledge, the present study represents the most comprehensive examination of the development and cumulative outcomes of comorbidity between MDD and AUD provided by a prospective study. Our study confirms the significant comorbidity between mood and alcohol use disorders reported (1-3), but extends previous findings in several important ways. First, we find considerably higher lifetime prevalence rates of MDD+AUD than previous epidemiological studies. One out of five participants reported a history of comorbidity by age 30 , compared to much lower rates in other studies (e.g., 4\%) (18). This difference is likely to mostly reflect under-reporting of disorder rates in retrospective studies (31), but could also be related to the use of full review of past periods between assessments, the use of semistructured rather than structured interviews, and K-SADS and SCID definitions for MDD and AUD symptom thresholds. Although the OADP population may over-represent White populations, multiple assessements combined with full coverage of past periods is likely to provide amongst the most accurate picture of cumulative disorder rates as can be found in available representative prospective samples (32). Second, we find that by age 30, comorbidity represents the majority of cases in women with a history of AUD and men with a history of MDD, as well as approximately half of cases in men with a history of AUD. Clearly, comorbidity is normative rather than an exception in individuals with either disorder, except for women with a history of MDD. Third, we found comorbidity to be rare in adolescence (mostly due to low rates of AUD), but already elevated by early adulthood. Rates of period comorbidity were highest in early adulthood and the majority of individuals with AUD already had a history of MDD by that time. Fourth, we found roughly equal lifetime rates of concurrent and successive comorbidity by age 30. In most cases, concurrent episodes developed after remission from non-concurrent episode(s) of MDD, AUD, or both. This novel finding suggests that concurrency tends to occur at a later stage in a trajectory of successive comorbidity.

A key focus of this study was on the relation between MDD and AUD over time. We first examined temporal ordering. Consistent with previous studies $(1,7,8)$, we found that MDD tended to occur prior to AUD ( $57 \%$ of lifetime cases). Only $2 \%$ of lifetime cases had a simultaneous onset of first incidence MDD and AUD, reinforcing the notion of concurrent comorbidity as an end-stage phenomenon. Interestingly, we found the converse pattern when examining temporal ordering in concurrent episodes specifically: AUD tended to occur first in the majority of concurrent episodes (57\%) and MDD occurred first in only $23 \%$ of concurrent episodes. We also examined prospective associations between MDD and AUD over time. We jointly considered cross-sectional and prospective associations across the three developmental periods, adjusting for disorder stability over time and potential psychiatric confounds. We found AUD in adolescence to predict MDD in early adulthood, but not the reverse. Conversely, we found MDD in early adulthood to predict AUD in adulthood, but not the reverse.

Taken together, these findings suggest that MDD and AUD relate in a complex manner. Multiple explanations have been proposed to explain comorbidity between heterotypic psychiatric disorders (6): 1) comorbidity represents a marker for the severity of relatively undifferentiated symptoms, 2) one disorder is a manifestation of the other, 3) one disorder 
causes the other, 4) two disorders share common causes, and 5) comorbidity is a marker for a unique disorder subtype. The present results suggest that several mechanisms may apply to comorbid MDD+AUD. The greater temporal precedence of MDD in individuals with lifetime MDD+AUD is consistent with a potential causal effect of MDD on AUD, but does not preclude other explanations. For instance, as MDD and AUD relate to separate but correlated liabilities for Internalizing and Externalizing traits respectively (33), shared vulnerability for these two traits could explain why the disorders are linked despite emerging at different times. On the other hand, the greater temporal precedence of AUD in concurrent episodes may reflect different etiologic processes. This pattern is consistent with a proximal causal effect of AUD on the development of MDD, but could also index some MDD episodes as manifestations of AUD or a subtype of alcohol-induced MDD (34).

Prospective associations across developmental periods are the strongest evidence in favor of a causal relation between MDD and AUD. Our findings suggest modest, bidirectional, and developmentally-specific associations between the two disorders. This finding is broadly consistent with studies showing AUD to predict MDD $(12,13)$, MDD to predict AUD $(8$, $11)$ or both $(9,14,15)$. Mixed results from previous studies could be partly due to a failure to account for developmental timing. Adolescent AUD may contribute to MDD risk in early adulthood by bringing about negative psychosocial consequences that are developmentally salient as adolescents move into adulthood (e.g. academic difficulties). Conversely, early adult MDD may contribute to the risk of AUD in adulthood via a "self-medication" mechanism (35) emerging specifically as individuals mature. However, it should be noted that self-medication is most consistent with AUD occurring after MDD in concurrent episodes as an attempt to cope with depressive symptoms, a pattern which we did not frequently observe. This raises the possibility that a mechanism other than self-medication mediates long-term associations.

Another focus of this study was on sequala of cumulative comorbidity. Contrary to expectation, we did not find lifetime MDD+AUD to be broadly associated with more clinical and psychosocial impairments than pure disorders. Nonetheless, we did identify several key negative correlates of comorbidity. We confirm the well-established finding (4, 18) that comorbidity increases the risk of suicide attempts, especially in individuals with AUD. Comorbidity also more than doubled the risk of alcohol severity, as defined by the presence of alcohol dependence, compared to pure AUD. This result is consistent with recent studies showing stronger comorbidity between MDD and alcohol dependence than abuse $(1,3,15)$. Although the two alcohol use disorders are merged into a single diagnosis in DSM-5 (24), our findings paired with previous research suggest that alcohol dependence symptoms should remain a valuable marker in research on comorbidity with depression. Also consistent with previous studies (4), we found comorbidity to be associated with poorer functioning and life satisfaction than pure MDD and AUD.

Several interesting differences between comorbid subtypes emerged in terms of clinical characteristics and psychosocial outcomes. Like others (18), we found MDD-first comorbidity to be associated with increased depression duration and suicidality compared to other comorbid groups and pure MDD. However, MDD duration was specifically increased when MDD-first was paired with concurrent comorbidity, with increased duration likely 
reflecting more MDD episodes in concurrent (mean=2.9) vs. other comorbid subtypes (means=1.8-2.0). One possibility is that a subset of individuals with a history of recurrent and more chronic depression are at risk of eventually developing an alcohol use disorder, which may represent an unsuccessful attempt to cope with mood symptoms that serves to maintain or exacerbate depression over time. Successive comorbidity was also associated with fewer problematic outcomes (functioning, risky sexual behaviors, AUD duration) than concurrent comorbidity.

\section{Limitations}

Study limitations should be noted. First, we combined diagnoses of alcohol abuse and dependence for most analyses. This approach did not allow us to investigate the specific importance of dependence symptoms $(1,3,15)$ and use of a broad definition may increase the possibility of conflicting findings across studies (36). On the other hand, our approach is consistent with DSM-5 (24) and is supported by the facts that alcohol abuse is hard to diagnose reliably and that diagnoses of abuse and dependence tend to form a single latent dimension (37). Another justification for examining a single diagnostic category is that more than a quarter (28\%) of individuals in the AUD group had a history of both diagnoses by age 30. Second, our design was not fully prospective. Using prospective assessments paired with recall allowed us to have full diagnostic coverage from adolescence to adulthood, but this strategy still involved a degree of recall bias. Third, the OADP dataset is not fully representative and particularly over-represents White participants. Fourth, we used OADP weights to adjust for missing data by design, but did not correct for potential attrition bias using approaches such multiple imputation or maximum likelihood estimation.

\section{Implications}

Several directions for future research can be suggested from this study. More prospective studies are needed, especially research examining risk factors (e.g., temperamental dispositions, early life experiences, neurobiological characteristics) that prospectively anticipate comorbid MDD+AUD. Theoretically, etiological models on MDD+AUD should be refined to take into account developmental factors, including concurrency and developmental timing. With regards to nosological models, our findings on concurrent disorders support the potential relevance of an alcohol-induced depression subtype.

The present findings also have important implications for clinical practice with both adolescents and adults. Our research re-emphasizes that treatment should always start with a comprehensive assessment of current and past psychopathology. As most adults with MDD or AUD will have a history of other disorder (except perhaps women with MDD), clinicians should inquire about both disorders with a special attention for symptoms of alcohol dependence, even under the new DSM-5. Treatment recommendations exist and should be used when addressing concurrent comorbidity (38). Whether and how treatment should be modified in cases of successive comorbidity is an understudied area that requires further attention. The developmental associations between MDD and AUD emphasize the importance of developmental timing in prevention. Intervening in AUD during adolescence may help to prevent future MDD and intervening in MDD early adulthood may help to prevent subsequent AUD. 


\section{Acknowledgments}

The authors wish to thank Jeff Gau and Derek B. Kosty for providing help with data organization and analysis. The authors have no conflict of interests to disclose. This study was supported by grants from the National Institute of Mental Health (MH40501, MH, 50522, and MH52858) and the National Institute on Drug Abuse (DA12951) awarded to Peter M. Lewinsohn, a grant from the National Institute on Alcohol Abuse and Alcoholism (AA020968) awarded to Richard F. Farmer and John R. Seeley, and a postdoctoral fellowship from the Fond Québécois de Recherche sur la Santé et la Société (FQRSC) awarded to Frédéric N. Brière. Funding organizations played no role in design and conduct of the study; collection, management, analysis, and interpretation of the data; and preparation, review, or approval of the manuscript. Authors had full access to data, are responsible for its integrity, and accuracy of analysis.

\section{References}

1. Boschloo L, Vogelzangs N, Smit JH, van den Brink W, Veltman DJ, Beekman AT, et al. Comorbidity and risk indicators for alcohol use disorders among persons with anxiety and/or depressive disorders: findings from the Netherlands Study of Depression and Anxiety (NESDA). J Affect Disord. 2011; 131(1-3):233-42. [PubMed: 21247636]

2. Grant BF, Harford TC. Comorbidity between DSM-IV alcohol use disorders and major depression: results of a national survey. Drug Alcohol Depend. 1995; 39(3):197-206. [PubMed: 8556968]

3. Hasin DS, Stinson FS, Ogburn E, Grant BF. Prevalence, correlates, disability, and comorbidity of DSM-IV alcohol abuse and dependence in the United States: results from the National Epidemiologic Survey on Alcohol and Related Conditions. Arch Gen Psychiatry. 2007; 64(7):83042. [PubMed: 17606817]

4. Cornelius JR, Salloum IM, Mezzich J, Cornelius MD, Fabrega H Jr, Ehler JG, et al. Disproportionate suicidality in patients with comorbid major depression and alcoholism. Am J Psychiatry. 1995; 152(3):358-64. [PubMed: 7864260]

5. Schuckit MA. The clinical implications of primary diagnostic groups among alcoholics. Arch Gen Psychiatry. 1985; 42(11):1043-9. [PubMed: 4051681]

6. Angold A, Costello EJ, Erkanli A. Comorbidity. J Child Psychol Psychiatry. 1999; 40(1):57-87. [PubMed: 10102726]

7. Dawson DA, Grant BF. Family history of alcoholism and gender: their combined effects on DSMIV alcohol dependence and major depression. J Stud Alcohol. 1998; 59(1):97-106. [PubMed: 9498321]

8. Kuo PH, Gardner CO, Kendler KS, Prescott CA. The temporal relationship of the onsets of alcohol dependence and major depression: using a genetically informative study design. Psychol Med. 2006; 36(8):1153-62. [PubMed: 16734951]

9. Flensborg-Madsen T, Mortensen EL, Knop J, Becker U, Sher L, Gronbaek M. Comorbidity and temporal ordering of alcohol use disorders and other psychiatric disorders: results from a Danish register-based study. Compr Psychiatry. 2009; 50(4):307-14. [PubMed: 19486728]

10. Merikangas KR, Mehta RL, Molnar BE, Walters EE, Swendsen JD, Aguilar-Gaziola S, et al. Comorbidity of substance use disorders with mood and anxiety disorders: results of the International Consortium in Psychiatric Epidemiology. Addict Behav. 1998; 23(6):893-907. [PubMed: 9801724]

11. McCarty CA, Kosterman R, Mason WA, McCauley E, Hawkins JD, Herrenkohl TI, et al. Longitudinal associations among depression, obesity and alcohol use disorders in young adulthood. Gen Hosp Psychiatry. 2009; 31(5):442-50. [PubMed: 19703638]

12. Fergusson DM, Boden JM, Horwood LJ. Tests of causal links between alcohol abuse or dependence and major depression. Arch Gen Psychiatry. 2009; 66(3):260-6. [PubMed: 19255375]

13. Rohde P, Lewinsohn PM, Kahler CW, Seeley JR, Brown RA. Natural course of alcohol use disorders from adolescence to young adulthood. J Am Acad Child Adolesc Psychiatry. 2001; 40(1):83-90. [PubMed: 11195569]

14. Gilman SE, Abraham HD. A longitudinal study of the order of onset of alcohol dependence and major depression. Drug Alcohol Depend. 2001; 63(3):277-86. [PubMed: 11418232] 
15. Bulloch A, Lavorato D, Williams J, Patten S. Alcohol consumption and major depression in the general population: the critical importance of dependence. Depress Anxiety. 2012; 29(12):105864. [PubMed: 22987574]

16. Grant BF, Goldstein RB, Chou SP, Huang B, Stinson FS, Dawson DA, et al. Sociodemographic and psychopathologic predictors of first incidence of DSM-IV substance use, mood and anxiety disorders: results from the Wave 2 National Epidemiologic Survey on Alcohol and Related Conditions. Mol Psychiatry. 2009; 14(11):1051-66. [PubMed: 18427559]

17. Moffitt TE, Harrington H, Caspi A, Kim-Cohen J, Goldberg D, Gregory AM, et al. Depression and generalized anxiety disorder: cumulative and sequential comorbidity in a birth cohort followed prospectively to age 32 years. Arch Gen Psychiatry. 2007; 64(6):651-60. [PubMed: 17548747]

18. Grant BF, Hasin DS, Dawson DA. The relationship between DSM-IV alcohol use disorders and DSM-IV major depression: examination of the primary-secondary distinction in a general population sample. J Affect Disord. 1996; 38(2-3):113-28. [PubMed: 8791180]

19. Mueller TI, Lavori PW, Keller MB, Swartz A, Warshaw M, Hasin D, et al. Prognostic effect of the variable course of alcoholism on the 10-year course of depression. Am J Psychiatry. 1994; 151(5): 701-6. [PubMed: 8166311]

20. Salloum IM, Mezzich JE, Cornelius J, Day NL, Daley D, Kirisci L. Clinical profile of comorbid major depression and alcohol use disorders in an initial psychiatric evaluation. Compr Psychiatry. 1995; 36(4):260-6. [PubMed: 7554869]

21. Davidson KM, Blackburn IM. Co-morbid depression and drinking outcome in those with alcohol dependence. Alcohol Alcohol. 1998; 33(5):482-7. [PubMed: 9811200]

22. Kranzler HR, Del Boca FK, Rounsaville BJ. Comorbid psychiatric diagnosis predicts three-year outcomes in alcoholics: a posttreatment natural history study. J Stud Alcohol. 1996; 57(6):619-26. [PubMed: 8913993]

23. Lewinsohn PM, Hops H, Roberts RE, Seeley JR, Andrews JA. Adolescent psychopathology: I. Prevalence and incidence of depression and other DSM-III-R disorders in high school students. J Abnorm Psychol. 1993; 102(1):133-44. Epub 1993/02/01. [PubMed: 8436689]

24. American Psychiatric Association. Diagnostic and statistical manual of mental disorders. 5th. Washington, DC: American psychiatric publishing; 2013.

25. Rohde P, Lewinsohn PM, Seeley JR, Klein DN, Andrews JA, Small JW. Psychosocial functioning of adults who experienced substance use disorders as adolescents. Psychol Addict Behav. 2007; 21(2):155-64. [PubMed: 17563135]

26. Orvaschel H, Puig-Antich J, Chambers W, Tabrizi MA, Johnson R. Retrospective assessment of prepubertal major depression with the Kiddie-SADS-e. J Am Acad Child Adolesc Psychiatry. 1982; 21(4):392-7.

27. First, MB.; Spitzer, RL.; Gibbon, M.; Williams, JBW. User's guide for the Structured Clinical Interview DSM-IV Axis I Disorders - Clinician version (SCID-I). Washington, DC: American Psychiatric Press; 1997.

28. Keller MB, Lavori PW, Friedman B, Nielsen E, Endicott J, McDonald-Scott P, et al. The Longitudinal Interval Follow-up Evaluation. A comprehensive method for assessing outcome in prospective longitudinal studies. Arch Gen Psychiatry. 1987; 44(6):540-8. [PubMed: 3579500]

29. Muthén, LK.; Muthén, BO. Mplus User's Guide. Sixth. Los Angeles, CA: Muthén \& Muthén;; 1998-2010.

30. McDonald RP, Ho MH. Principles and practice in reporting structural equation analyses. Psychol Methods. 2002; 7(1):64-82. [PubMed: 11928891]

31. Moffitt TE, Caspi A, Taylor A, Kokaua J, Milne BJ, Polanczyk G, et al. How common are common mental disorders? Evidence that lifetime prevalence rates are doubled by prospective versus retrospective ascertainment. Psychol Med. 2010; 40(6):899-909. [PubMed: 19719899]

32. Rohde P, Lewinsohn PM, Klein DN, Seeley JR, Gau JM. Key Characteristics of Major Depressive Disorder Occurring in Childhood, Adolescence, Emerging Adulthood, and Adulthood. Clin Psychol Science. 2013; 1(1):41-53.

33. Krueger RF. The structure of common mental disorders. Arch Gen Psychiatry. 1999; 56(10):9216. [PubMed: 10530634] 
34. Schuckit MA. Comorbidity between substance use disorders and psychiatric conditions. Addiction. 2006; 101 Suppl 1:76-88. [PubMed: 16930163]

35. Khantzian EJ. The self-medication hypothesis of addictive disorders: focus on heroin and cocaine dependence. Am J Psychiatry. 1985; 142(11):1259-64. [PubMed: 3904487]

36. Boschloo L, Vogelzangs N, van den Brink W, Smit JH, Veltman DJ, Beekman AT, et al. Alcohol use disorders and the course of depressive and anxiety disorders. Br J Psychiatry. 2012; 200(6): 476-84. [PubMed: 22322459]

37. Hasin DS, Schuckit MA, Martin CS, Grant BF, Bucholz KK, Helzer JE. The validity of DSM-IV alcohol dependence: what do we know and what do we need to know? Alcohol Clin Exp Res. 2003; 27(2):244-52. [PubMed: 12605073]

38. Kelly TM, Daley DC, Douaihy AB. Treatment of substance abusing patients with comorbid psychiatric disorders. Addict Behav. 2012; 37(1):11-24. [PubMed: 21981788] 


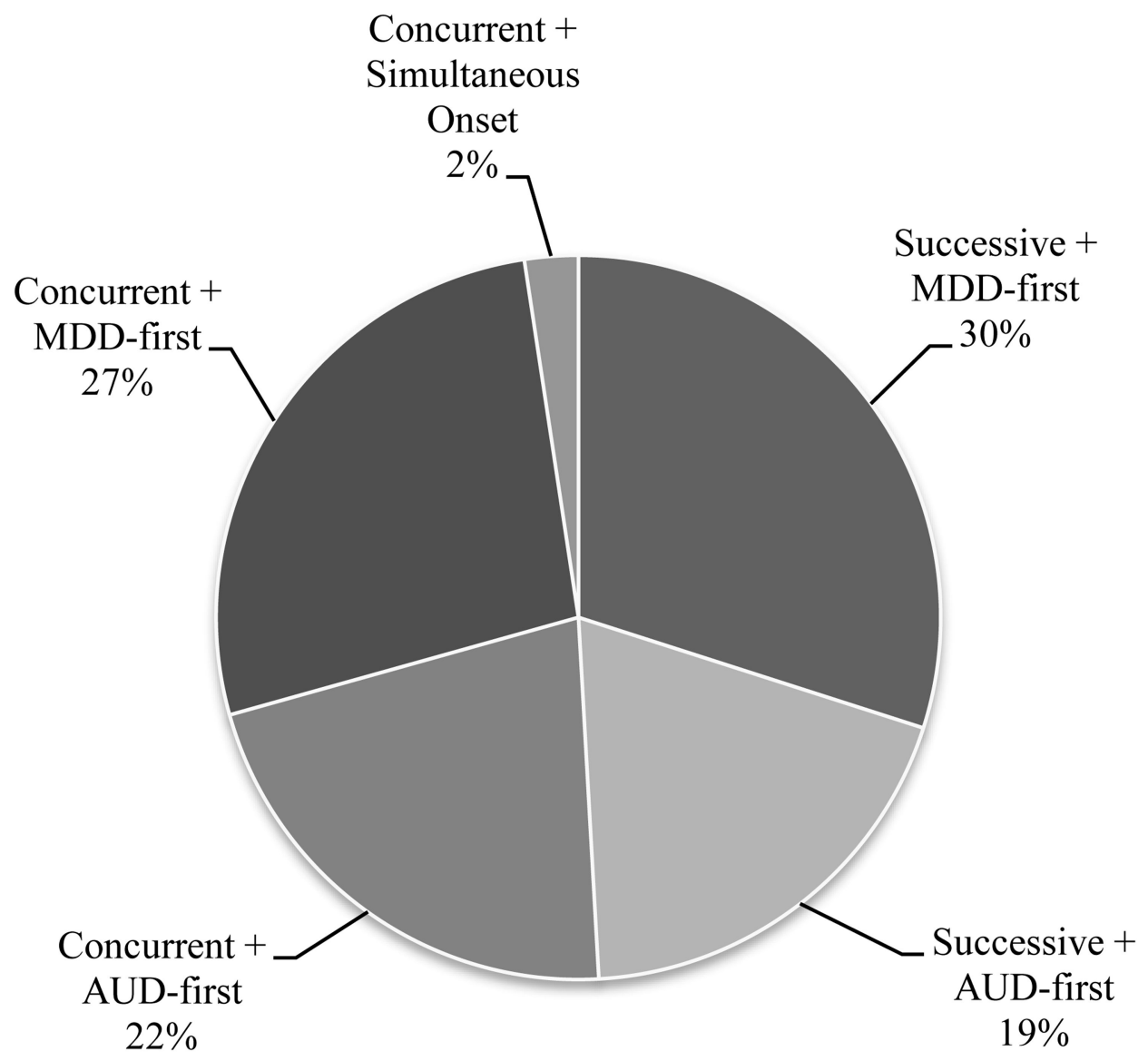

Figure 1. Subtypes of Cumulative Lifetime Comorbidity between MDD and AUD as a Function of Concurrency and Temporal Ordering 


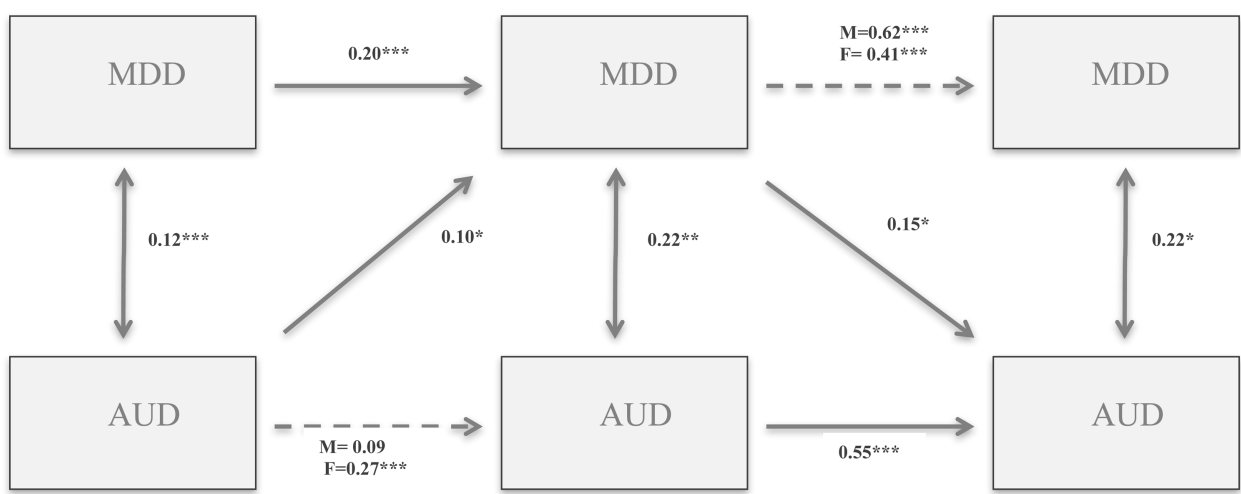

Figure 2. Overall and Gender-Specific Associations between MDD and AUD from Adolescence to Adulthood

Model fit: $\mathrm{CFI}=0.982 ; \mathrm{TLI}=982 ; \mathrm{RMSEA}=0.022$. Associations are standardized regression coefficients. Full lines represent gender invariant associations. Dashed arrows represent gender specific associations. The model adjusts for anxiety disorders, disruptive behavior disorders, and other substance use disorders in adolescence (not shown). Controls were specified to predict MDD and AUD in early adulthood and adulthood and to correlate together and with MDD and AUD in adolescence. 
Table 1

Weighted Period and Cumulative Prevalence of Pure and Comorbid MDD and AUD

\begin{tabular}{|c|c|c|c|c|}
\hline & & \multicolumn{3}{|c|}{ n $(\%)$} \\
\hline & & Pure MDD & Pure AUD & MDD+AUD \\
\hline \multicolumn{5}{|l|}{ Adolescence } \\
\hline & Women & $109(23.8)$ & $10(2.2)$ & $9(2.0)$ \\
\hline & Men & $33(9.2)$ & $16(4.5)$ & $6(1.7)$ \\
\hline & Total & $142(17.4)$ & $26(3.2)$ & $15(1.8)$ \\
\hline \multicolumn{5}{|c|}{ Early Adulthood } \\
\hline & Women & $134(29.3)$ & $48(10.5)$ & $48(10.5)$ \\
\hline & Men & $40(11.2)$ & 77 (21.5) & $39(10.9)$ \\
\hline & Total & $174(21.3)$ & $125(15.3)$ & 87 (10.7) \\
\hline \multicolumn{5}{|l|}{ Adulthood } \\
\hline & Women & $127(27.8)$ & $25(5.5)$ & $36(7.9)$ \\
\hline & Men & $35(9.8)$ & 63 (17.6) & $19(5.3)$ \\
\hline & Total & $162(19.8)$ & 89 (10.9) & $55(6.7)$ \\
\hline \multicolumn{5}{|l|}{ Lifetime } \\
\hline & Women & $203(44.3)$ & $34(7.3)$ & 94 (20.5) \\
\hline & Men & $51(14.2)$ & $81(22.8)$ & 74 (20.6) \\
\hline & Total & $254(31.1)$ & $115(14.1)$ & $167(20.5)$ \\
\hline
\end{tabular}


